\title{
The role of signal averaged $P$ wave duration and serum magnesium as a combined predictor of atrial fibrillation after elective coronary artery bypass surgery
}

\author{
A G Zaman, F Alamgir, T Richens, R Williams, M T Rothman, P G Mills
}

\begin{abstract}
Objective-To investigate the role of low serum magnesium as a trigger for atrial fibrillation in patients with a substrate for the arrhythmia (assessed by signal averaged $P$ wave duration).

Design-A case-control study.

Setting-A regional referral cardiac centre.

Patients and interventions-105 consecutive patients undergoing elective coronary artery bypass surgery had signal averaged $P$ wave recordings before operation. Serum electrolytes were analysed preoperatively and on days 1,2 , and 5 after surgery.

Main outcome measures-Any episode of electrocardiographically recorded atrial fibrillation was taken as a study end point. Results-Of 102 patients discharged, 27 (26\%) had documented episodes of atrial fibrillation at a mean of 2.7 days after surgery. A combination of $P$ wave duration $>155 \mathrm{~ms}$ and serum magnesium on the first postoperative day of $<0.7 \mathrm{mmol} / \mathrm{l}$ had a sensitivity of $75 \%$ and specificity of $80 \%$ for predicting atrial fibrillation. Duration of hospital stay (7.9 $v 6.8$ days) was longer in the atrial fibrillation group $(P<0 \cdot 01)$. Stepwise regression showed age, serum magnesium $<0.7 \mathrm{mmol} / 1$ on the first postoperative day (both $P<$ 0.001 , angiotensin converting enzyme inhibitor withdrawal $(P<0.02)$, and signal averaged $P$ wave duration $(P=0.04)$ to be independent predictors.

Conclusions-The combination of signal averaged $P$ wave duration and low serum magnesium on the first postoperative day identified the majority of patients with atrial fibrillation after coronary artery bypass surgery. Early identification and pharmacological treatment for selected patients may reduce the incidence of postoperative atrial fibrillation.
\end{abstract}

(Heart 1997;77:527-531)

Keywords: atrial fibrillation; coronary artery bypass surgery; signal averaging; magnesium

Coronary artery bypass surgery is well tolerated and for the majority of patients is free from complications. Among the most commonly encountered postoperative complications is atrial fibrillation. Its incidence has been reported at between $5 \%$ and $40 \%$ after cardiac surgery. ${ }^{1-6}$ It occurs in the first week, with a peak incidence on the second postoperative day. In most cases this arrhythmia is benign and self limiting. However, it may result in haemodynamic compromise, thromboemboli, and prolongation of hospital stay. ${ }^{3578}$ Clinical features which have often been identified as predictors of atrial fibrillation include advancing age and postoperative withdrawal of $\beta$ blocking drugs. ${ }^{34}$ In addition, prolonged $P$ wave duration on the high resolution electrocardiogram (ECG), indicating delayed atrial conduction, ${ }^{9}$ and the presence of severe right coronary artery stenosis ${ }^{10}$ have been implicated.

Magnesium has been shown to suppress all cardiac arrhythmias after acute myocardial infarction. ${ }^{11} 12$ In addition there are reports that low serum magnesium is associated with perioperative atrial fibrillation,,$^{13}$ and of magnesium supplementation in the treatment of supraventricular arrhythmias. ${ }^{14} \mathrm{~A}$ study by Parikka and coworkers, however, reported that high plasma magnesium concentrations perioperatively led to a higher incidence of postoperative atrial fibrillation. ${ }^{15}$

There are no studies examining the role of magnesium in patients selected on the basis of delayed atrial conduction. In order to assess this, we investigated the role of serum magnesium as a trigger for atrial fibrillation in patients with the arrhythmia substrate, as identified by prolonged $\mathrm{P}$ wave conduction on high resolution ECG.

\section{Methods}

One hundred and five consecutive patients undergoing their first elective coronary artery bypass graft surgery were enrolled over a four month period. We excluded patients in atrial fibrillation or those with a past history of atrial fibrillation, patients on class I or III antiarrhythmic drugs (which cause prolongation of atrial conduction), and patients undergoing valve surgery.

\section{CLINICAL EVALUATION}

All patients were nursed initially in the cardiac intensive care unit and then on the cardiothoracic wards until discharge. All were monitored for the first 48 hours on ECG telemetry with a central monitor at the nursing station. A 12lead ECG was recorded whenever a change in rhythm was seen on the monitor. Nursing 
observations were performed routinely every four hours and 12-lead ECGs recorded whenever a cardiac dysrhythmia was suspected. Every recorded ECG was reviewed by one of us (AGZ). All patients had routine 12-lead ECGs on days $1,2,3,5$, and on the day of discharge. In addition, ECGs were recorded if there was clinical suspicion of a cardiac dysrhythmia.

Any documented episode of atrial fibrillation on a 12-lead ECG was defined as a study end point. The postoperative day when atrial fibrillation started was recorded. Hospital stay was defined as the number of days of inpatient care from the day of surgery. All angiograms were read by an experienced invasive cardiologist.

Preoperative variables recorded were age, sex, medication, presence of critical right coronary artery stenosis (defined as narrowing of the proximal or mid-segment greater than or equal to $70 \%$ of lumen diameter), left ventricular hypertrophy (assessed on preoperative ECG), left ventricular ejection fraction (assessed on biplane cine ventriculogram), and $P$ wave duration on the high resolution ECG. Intraoperative variables included operator status, aortic cross clamp time, pump time, use of cardioplegia, and number of grafts. Postoperatively, the total amount of blood transfused was recorded.

\section{BIOCHEMICAL ANALYSES}

Serum magnesium and potassium were measured preoperatively and on days 1,2 , and 5 after surgery. Serum magnesium was measured by spectrophotometry using the zylidyl blue method (Synermed, Monitor Bioscience, West Sussex, UK). Serum potassium was measured by ion selective electrode on a Dax 48 analyser (Bayer, Hampshire, UK). The reference range for serum magnesium in our laboratory is $0 \cdot 7-1 \cdot 00 \mathrm{mmol} / \mathrm{l}$

HIGH RESOLUTION ECG

The method of recording and analysing $P$ wave signal averaged ECG has been described before. ${ }^{16}$ All high resolution ECGs were recorded by a qualified cardiac technician. After careful preparation of the skin, silver-silver chloride electrodes were applied in an orthogonal arrangement. The QRS was used as the trigger for the signal averaging process. In order to expose the $\mathrm{P}$ wave and PR segment, the fiducial point was shifted to the extreme right of the $300 \mathrm{~ms}$ window. A P wave template was manually selected and $P$ wave complexes not matching the template with a $99 \%$ correla- tion coefficient were automatically rejected. This ensured that any variation in the $P$ wave complex due to surface noise or to ectopic or autonomic activity was not averaged. The $\mathrm{P}$ waves were acquired until a noise end point of $<0.5 \mu \mathrm{V}$. If this required more than 500 beats, the recording was repeated until the criterion was met. A least squares fit filter with a window width of $100 \mathrm{~ms}$ (high pass cut off of $29 \mathrm{~Hz}$ ) was applied for data analysis. The $P$ wave complexes of the $\mathrm{X}, \mathrm{Y}$, and $\mathrm{Z}$ bipolar leads were combined into a vector magnitude calculated using the root mean square of each lead.

The $P$ wave onset and offset were determined manually and $P$ wave duration calculated preoperatively (by AGZ). The onset was defined as the first atrial deflection from the baseline noise level and offset as the return of the atrial signal to baseline or onset of the QRS complex, whichever was earlier. ${ }^{16}$

\section{SURGICAL PROCEDURE}

Coronary artery bypass surgery was performed by a consultant surgeon or senior registrar or by a registrar with a consultant in attendance. The status of the operator was recorded. After initiation of cardiopulmonary bypass, myocardial protection was achieved either with cooling to $28^{\circ} \mathrm{C}$ and cold crystalloid cardioplegia, or with cross clamp fibrillation and a temperature of $32^{\circ} \mathrm{C}$. Conduits for bypass included the saphenous veins or internal mammary artery (whenever possible) or a combination of the two.

\section{STATISTICAL ANALYSIS}

Results are presented as mean (SD). Initial comparisons using univariate analysis between groups were made (Student $t$ test for continuous variables and $\chi^{2}$ or Fisher's exact test where appropriate, for categorical variables).

Stepwise logistic regression analysis was performed on variables found to be significant predictors with univariate analysis.

The variables considered were age, sex, serum preoperative and postoperative magnesium, potassium and haemoglobin, $\beta$ blocker withdrawal, use of calcium antagonist, angiotensin converting enzyme (ACE) inhibitors and diuretics, critical right artery stenosis, left ventricular ejection fraction, signal averaged $P$ wave duration, aortic cross clamp time, use of cardioplegia, pump time, need for blood transfusion, and status of operator.

A $P$ value of $<0.05$ was considered statistically significant.

\section{Results}

Of the 105 patients studied, three died before hospital discharge, one from myocardial infarction and two from pump failure. None had documented atrial fibrillation.

Of the remaining 102 patients discharged from hospital, $27(26 \%)$ had episodes of atrial fibrillation recorded on at least one 12-lead ECG. Of these, two reverted to sinus rhythm in less than 24 hours and six were in atrial fibrillation on discharge. Twenty three patients received antiarrhythmic medication and four 
Table 2 Univariate analysis of predictors of atrial fibrillation after coronary artery bypass surgery

\begin{tabular}{ll}
\hline Variable & Pvalue \\
\hline Serum magnesium (day 1) & $<0.001$ \\
Age & $<0.001$ \\
Signal averaged P wave duration & $<0.01$ \\
ACE inhibitor withdrawal & 0.02 \\
$\beta$ blocker withdrawal & 0.04 \\
\hline
\end{tabular}

$\mathrm{ACE}$, angiotensin converting enzyme.

Figure 1 Change in serum magnesium after surgery in patients with and without atrial fibrillation. The difference between the two groups on the first postoperative day is statistically significant. ${ }^{\star} P<0.001$

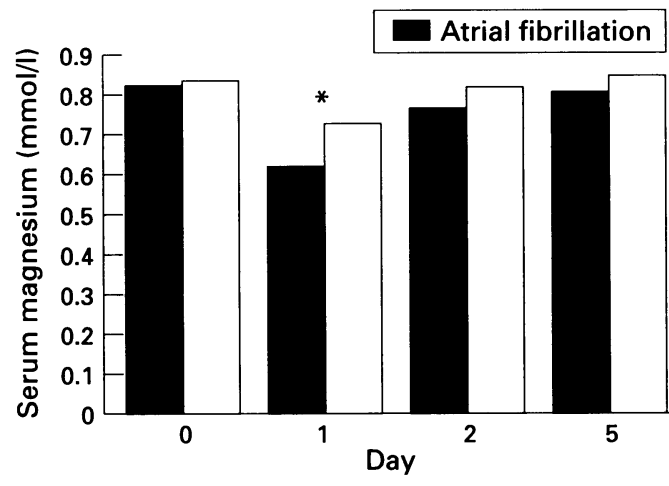

Figure 2 Change in serum potassium after surgery in patients with and without atrial fibrillation.

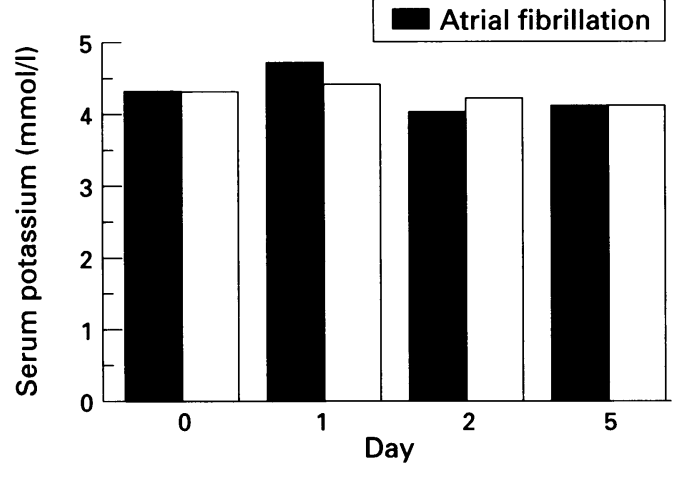

were cardioverted before discharge. Seven patients had missing values for serum magnesium because of haemolysis. None of these patients had recorded atrial fibrillation.

Atrial fibrillation occurred at a mean of 2.7 days (range 1-9) after coronary artery bypass surgery. The clinical characteristics of the patients are compared in table 1.

Of the variables tested by univariate analysis, serum magnesium on the first postoperative day, advancing age, $\beta$ blocker and ACE inhibitor usage, and $P$ wave duration were associated with atrial fibrillation.

The serum magnesium values, together with the other significant predictors on univariate analysis, are shown in table 2 , in which patients are stratified according to the presence of atrial fibrillation. Patients with the arrhythmia were significantly older and had longer $\mathbf{P}$ wave duration on the signal averaged ECG. $\beta$ blocker withdrawal was only marginally significant as a predictor. Surprisingly the withdrawal of preoperative angiotensin-converting enzyme (ACE) inhibition (13 patients) was a stronger predictor of postoperative atrial

Table 3 Sensitivity, specificity, and positive predictive accuracy of selected variables

\begin{tabular}{llll}
\hline & $\begin{array}{l}M g<0.7 \text { mmolll and } \\
P \text { wave }>155 \mathrm{~ms}(\%)\end{array}$ & $\begin{array}{l}M g<0.7 \\
\text { mmoll/ (\%) }\end{array}$ & $\begin{array}{l}P \text { wave }>155 \\
\text { ms (\%) }\end{array}$ \\
\hline Sensitivity & 75 & 85 & 86 \\
Specificity & 80 & 58 & 45 \\
Positive predictive accuracy & 62 & 45 & 37 \\
\hline
\end{tabular}

fibrillation.

Hospital stay was longer in the atrial fibrillation group $(7.9 v 6.8$ days; $P<0.01)$. Of the other clinical, angiographic, and haemodynamic variables, none was predictive of postoperative atrial fibrillation.

\section{SERUM MAGNESIUM AND POTASSIUM}

For the group as a whole, serum magnesium fell on the first postoperative day (from 0.83 (SD 0.1$)$ to $0.69(0.1) \mathrm{mmol} / \mathrm{l} ; \mathrm{P}<0.001)$. The values increased on the second postoperative day to $0 \cdot 79(0 \cdot 1) \mathrm{mmol} / 1$ and had returned to preoperative levels by day $5(0.83(0 \cdot 1)$ $\mathrm{mmol} / \mathrm{l})$.

When the patients were divided on the basis of atrial fibrillation there was no difference in preoperative magnesium values: $0.82(0.1)$ $\mathrm{mmol} / 1$ in those with atrial fibrillation versus $0.83(0.1) \mathrm{mmol} / \mathrm{l}$ in those without. Postoperatively, however, there was a significant difference between the atrial fibrillation and sinus rhythm groups on the first postoperative day: $0.62(0.1)$ versus $0.72(0.1) \mathrm{mmol} / 1(P<$ $0 \cdot 001$ ), respectively (fig 1 ).

There was no significant difference in potassium values between the groups with or without atrial fibrillation. Preoperatively the value for both groups was $4.3 \mathrm{mmol} / 1$. On the first postoperative day the values were $4.4(0.4)$ and $4.5(0 \cdot 6) \mathrm{mmol} / \mathrm{l}$, respectively (fig 2$)$.

SIGNAL AVERAGED P WAVE DURATION

The signal averaged $P$ wave duration was significantly longer in patients with postoperative atrial fibrillation, at 165 (15) versus 155 (18) $\mathrm{ms}(\mathrm{P}<0.01)$.

\section{MULTIVARIATE ANALYSIS}

Stepwise logistic regression was performed for each of the variables that were found to be significant predictors on univariate analysis. This showed that advancing age and hypomagnesaemia on the first postoperative day were the most powerful predictors (both $P<0.001$ ). They were followed in predictive power by withdrawal of ACE inhibitors $(P=0.02)$ and $P$ wave duration $(P=0.04) ; \beta$ blocker withdrawal was no longer significant.

\section{SENSITIVITY AND SPECIFICITY OF P WAVE}

DURATION AND SERUM MAGNESIUM IN PREDICTING POSTOPERATIVE ATRIAL

FIBRILLATION

The sensitivity, specificity, and positive predictive accuracy for $P$ wave duration, serum magnesium on day 1 , and a combination of the two are presented in table 3 . A combination of $P$ wave duration and serum magnesium gave a sensitivity of $75 \%$ with a specificity of $80 \%$ and positive predictive accuracy of $62 \%$.

\section{Discussion}

The extensive literature on the subject of postoperative atrial fibrillation is testament to the increased length of stay and morbidity associated with the phenomenon. Major advances in coronary artery surgery have not been paralleled by a significant decline in postoperative 
atrial fibrillation, and it is likely that the cause is multifactorial. This study was designed to see if the signal averaged $P$ wave could be used as a marker for patients predisposed to develop atrial fibrillation after routine coronary artery bypass surgery. In addition, we hypothesised that hypomagnesaemia might be an important trigger in patients with this electrophysiological substrate.

Our study confirms the high incidence of atrial fibrillation after elective coronary artery bypass surgery (26\%). Age and low serum magnesium on the first postoperative day were powerful independent predictors of atrial fibrillation. Age is an important but unmodifiable factor. As anaesthetic and surgical techniques improve, increasing numbers of older patients are being offered coronary artery bypass surgery. It is possible therefore that the incidence reported will increase with time.

Postoperative atrial fibrillation was associated with longer hospital admission. Consistent with other studies, signal averaged $P$ wave duration was also predictive of atrial fibrillation.

A combination of preoperative signal averaged $P$ wave duration and hypomagnesaemia on the first postoperative day improves significantly on previous criteria for patient identification.

Although withdrawal of $\beta$ blockers was associated with atrial fibrillation, this was not an independent predictor. Somewhat surprisingly, the withdrawal of ACE inhibitors was an independent predictor. However, no firm conclusions about this can be drawn, since the use of ACE inhibitors in this patient population was small (11\%).

\section{SIGNAL AVERAGED P WAVE}

Prolongation of the $P$ wave duration has been shown to be associated with both paroxysmal and postoperative atrial fibrillation. ${ }^{91718}$ Atrial fibrillation is re-entrant in origin, with multiple continuous intra-atrial micro re-entrant circuits (the multiple wavelet hypothesis). ${ }^{19}$ One factor that can facilitate this arrhythmia is depressed intra-atrial conduction, which would manifest as lengthening of the $P$ wave recorded on the ECG. The signal averaged ECG can enhance detection of conduction delay within the atria, especially if it occurs late in relatively small portions of the atria..$^{9}$ In our study the $\mathrm{P}$ wave duration measured on the signal averaged ECG was an independent predictor of postoperative atrial fibrillation. It was a more powerful predictor than most other clinical variables but weaker than hypomagnesaemia and age.

A prolonged preoperative $P$ wave duration suggests that patients who develop postoperative atrial fibrillation have an intrinsic atrial electrical abnormality predisposing to this arrhythmia. However, atrial fibrillation had not occurred in these patients before the operation. It is likely therefore that delayed atrial activation is merely a marker for the arrhythmia substrate. Hypomagnesaemia or other stresses resulting from the operation may act as a trigger for the arrhythmia.
POSTOPERATIVE HYPOMAGNESAEMIA

The role of magnesium as a trigger for cardiac arrhythmias is well established. Most studies have been conducted in the setting of an acute myocardial infarction, ${ }^{112}$ and magnesium replacement has been shown to reduce the incidence of arrhythmias in this setting.

Our finding of a fall in serum magnesium after coronary artery bypass surgery confirms the results of Bunton's retrospective study of 200 patients. ${ }^{20}$ As with our prospective study, serum magnesium levels fell to a minimum on the first postoperative day, returning to their preoperative levels by the fourth day.

Vejlsted and Eliasen also reported postoperative hypomagnesaemia in a study with a smaller patient sample. ${ }^{21}$ Neither of these studies, however, examined the correlation between a fall in serum magnesium and postoperative complications or arrhythmias. There is evidence, however, of a reduction in the incidence of atrial fibrillation after coronary bypass surgery following magnesium replacement in an unselected group. ${ }^{22} 23$

Haemodilution, the use of diuretics, secondary hyperaldosteronism, and increased anabolic activity have been suggested as possible causes for the decline in serum magnesium after cardiac surgery. ${ }^{18}$ Hypomagnesaemia is also seen under conditions of acute stress and may be a result of enhanced sympathetic activity. ${ }^{24} 25$ There is experimental evidence for sequestration of magnesium in adipose tissue, ${ }^{26}$ but no evidence for an acute intracellular shift ${ }^{27}$ or increased urinary excretion of magnesium. ${ }^{1822}$

Although the positive predictive value of hypomagnesaemia was poor, when combined with $P$ wave duration the specificity and positive predictive value were significantly better. This improvement may in part be due to the fact that, whereas a prolonged $P$ wave identifies patients with the arrhythmia substrate, low serum magnesium identifies patients with one possible trigger for this arrhythmia.

A study by Parikka and co-workers ${ }^{15}$ found that giving magnesium replacement to an unselected group did not reduce the incidence of postoperative atrial fibrillation. This is consistent with our study, which shows that serum magnesium alone has low specificity and positive predictive value. It is only in combination with signal averaged $P$ wave duration that one can select a majority of patients who will develop the arrhythmia. Further work is required to look at the role of magnesium supplementation in patients selected on the basis of the above two variables.

Low serum potassium is often found in association with hypomagnesaemia and is known to be arrhythmogenic. ${ }^{28}$ In our study, however, serum potassium did not fall significantly after operation and there was no difference in potassium concentrations between the two groups.

POTENTIAL MECHANISMS FOR POSTOPERATIVE ATRIAL FIBRILLATION

The mechanism by which low serum magnesium may predispose to atrial fibrillation is unclear. Low magnesium perfusion has been shown to shorten the action potential duration 
and effective refractory period in rabbit ventricular muscle. ${ }^{29}$ Since the shortening of action potential duration at $90 \%$ repolarisation was greater than that of the effective refractory period, relative refractory period was prolonged and the ventricles became much more vulnerable to ventricular fibrillation. Although these investigators did not study magnesium effects on the atrial muscle, similar changes occurring in atrial fibres could well facilitate production of atrial fibrillation by a premature stimulus.

Extracellular magnesium alone has only minor effects on the action potential of cardiac cells unless the concentration of potassium is altered. ${ }^{29}{ }^{30}$ Hypomagnesaemia is considered to be arrhythmogenic because infusions of magnesium have terminated arrhythmias in hypomagnesaemic patients. $^{31}$ Hypomagnesaemia may, of course, be a confounding factor and merely reflect other stresses during the perioperative and postoperative periods. One other possibility is that it is the flux of magnesium ions in the perioperative period, rather than the absolute level, which acts as a trigger. If this were the case, then magnesium replacement may not affect the incidence of atrial fibrillation. The role of magnesium replacement in selected patients therefore requires further investigation. In some studies it has been shown to reduce the incidence of atrial fibrillation after coronary artery bypass surgery. ${ }^{20} 21$

\section{LIMITATIONS OF THE STUDY}

Telemetry data were available only for the first 48 hours in all cases. The majority of clinically important episodes occurred after this time. It is possible, therefore, that short lived episodes of atrial fibrillation not noted by the patient or medical staff were not diagnosed in this later postoperative period. This could lead to an overestimation of sensitivity and positive predictive values. However, our prevalence figure of $26 \%$ is similar to that reported in larger studies. ${ }^{14}$ In addition, the prolonged postoperative hospital stay noted in other published reports and in our study is likely to have been caused by clinically relevant episodes.

Although left atrial size and mitral regurgitation are recognised risk factors for the development of atrial fibrillation in the general population, this is not the case in patients after coronary artery bypass surgery. In a review of over 2400 patients by Mathew et $a l,{ }^{4}$ left atrial size was not associated with atrial fibrillation in this patient population. Routine preoperative echocardiograms are unlikely to be cost-effective in this clinical context and were not therefore routinely performed. In addition poor echogenicity may have been a problem in this patient population.

1 Lauer MS, Eagle KA, Buckley MJ, DeSanctis RW. Atrial fibrillation following coronary artery bypass surgery. Prog Cardiovasc Dis 1989;31:367-8.

2 Crosby L, Pifalo W, Woll K, Burkholder J. Risk factors for atrial fibrillation after coronary artery bypass grafting. $\mathrm{Am}$ f Cardiol 1990;66:1520-2.

3 Fuller JA, Adams GC, Buxton B. Atrial fibrillation after coronary artery bypass grafting: is it a disorder of the elderly? $\mathcal{F}$ Thorac Cardiovasc Surg 1989;97:821-5.

4 Mathew JP, Parks R, Savino JS, Friedman AS, Koch C, Mangano DT, et al. Atrial fibrillation following coronary artery bypass graft surgery. $\mathcal{Y} A M A 1996 ; 276: 300-6$

5 Rubin D, Mieminski K, Reed G, Herman M. Predictors, prevention, and long-term prognosis after coronary artery bypass graft operations. $\mathcal{F}$ Thorac Cardiovasc Surg 1987; 94:331-5.

6 Aranki SF, Shaw DP, Adams DH, Rizzo RJ, Couper GS VanderVliet $M$, et al. Predictors of atrial fibrillation after coronary artery bypass surgery: current trends and coronary artery bypass surgery: current trends and

7 Davison R, Hertz R, Kaplan K, Parker M, Feiercisel P, Michaelis $\mathrm{L}$ et al. Prophylaxis of supraventricular tachyarchyth $\mathrm{L}$, et al. Prophylaxis of supraventricular tachyarrhythmias after coronary artery bypass surgery with ora verapamil: a randomized,

8 Taylor GJ, Malik SA, Colliver JA, Dove JT, Moses HW, Mikell FL, et al. Usefulness of atrial fibrillation as a preMikell FL, et al. Usefulness of atrial fibrillation as a pre-
dictor of stroke after isolated coronary artery bypass grafting. Am $₹$ Cardiol 1987;60:905-7.

9 Steinberg JS, Zelenkofske S, Wong SC, Gelernt M, Sciacca $R$, Menchavez E. Value of the $P$-wave signal-averaged ECG for predicting atrial fibrillation after cardiac surgery. Circulation 1993;88:2618-22.

10 Mendes LA, Connelly GP, McKenney PA, Podrid PJ, Cupples A, Shemin RJ, et al. Right coronary artery stenosis: an independent predictor of atrial fibrillation afte coronary artery bypass surgery. $\mathcal{f}$ Am Coll Cardiol 1995; 25:198-202.

11 Rasmussen HS, Norregard P, Lindeneg O, McNair P, Backer V, Balslev $S$. Intravenous magnesium in acute myocardial infarction. Lancet 1986;i:234-6.

12 Abraham AS, Rosenmann D, Kramer M, Balkin J, Zion M Farbstein $\mathrm{H}$, et al. Magnesium in the prevention of lethal arrhythmias in acute myocardial infarction. Arch Intern Med 1987;147:753-5.

13 Ranasinghe DN, Mallett SV. Hypomagnesaemia, cardiac arrhythmias and orthotopic liver transplantation. arrhythmias and orthot

14 Gullestad L, Birkeland K, Molstad P, Hoyer MM, Vanber $P$, Kjekshus J. The effect of magnesium versus verapamil $\mathrm{P}$, Kjekshus J. The effect of magnesium versus verapamil on suprave

15 Parikka H, Toivonen L, Pellinen T, Verkkala K, Jarvinen A, Nieminen MS. The influence of intravenous magnesium sulphate on the occurrence of atrial fibrillation after coronary artery bypass operation. Eur Heart $\mathcal{F} 1993 ; 14$ 251-8

16 Guidera SA, Steinberg JS. The signal-averaged P-wave duration: a rapid and non-invasive marker of risk of atria fibrillation. $\mathcal{F}$ Am Coll Cardiol 1993;21:1645-51.

17 Fukunami M, Yamada $T$, Ohmori $M$, Kumagai $K$, Umemoto K, Sakai A, et al. Detection of patients at risk for paroxysmal atrial fibrillation during sinus rhythm by $P$-wave triggered signal-averaged electrocardiogram. Circulation 1991;83:162-9.

18 Stafford PJ, Turner I, Vincent R. Quantitative analysis of signal-averaged $\mathrm{P}$-waves in idiopathic paroxysmal atrial fibrillation. Am f Cardiol 1991;68:751-5.

19 Moe GK. On the multiple wavelet hypothesis of atrial fibrillation. Arch Int Pharmacodyn Ther 1962;140:183-8.

20 Bunton RW. Value of serum magnesium estimation in diagnosing myocardial infarction and predicting dysrhythmias after coronary artery bypass grafting. Thorax 1983;38:946-50.

21 Vejlsted H, Eliasen P. Postoperative serum level and urinary excretion of magnesium following heart surgery. Scand $\mathcal{F}$ Thorac Cardiovasc Surg 1978;12:91-4

22 Fanning WJ, Thomas CS, Roach A, Tomchek R, Alford WC, Stoney WS. Prophylaxis of atrial fibrillation with magnesium sulfate after coronary artery bypass grafting. Ann Thorac Surg 1991;53:529-33.

23 Colquhoun W, Berg GA, El-Fiky M, Fell GS, Wheatley DJ. Arrhythmia prophylaxis after coronary artery surgery. Eur $\mathcal{f}$ Cardiothorac Surg 1993;7:520-3

24 Whyte KF, Addis GJ, Whitesmith R, Reid JL. Adrenergic control of magnesium in man. Clin Sci 1987;72:135-8.

25 Rasmussen HS, Aurup P, Hojberg S, Kehn Jensen E McNair P. Transient hypomagnesaemia not induced by renal magnesium loss in patients with myocardial infarction. Arch Intern Med 1986;146:872-4.

26 Flink EB, Brick JE, Shane SR. Alterations of long-chain free fatty acid and magnesium concentrations in acute myorar

27 Ryzen E, Servis KI, Rude RK. Effect of intravenous epinephrine on serum magnesium and free intracellular red blood cell magnesium concentrations measured by nuclear magnetic resonance. $₹$ Am Coll Nutr 1990;9: 114-19.

28 Dyckner T, Wester PO. Ventricular extrasystoles and intracellular electrolytes before and after potassium and magnesium infusions in patients on diuretic treatment. $A m$ Heart $\mathcal{F}$ 1979;97:12-18.

29 Watanabe Y, Dreifus LS. Electrophysiological effects of magnesium and its interaction with potassium

30 Draft LF, Katholi RE, Woods WT, James TN. Attenuation by magnesium of the electrophysiologic effects of hyperkalaemia on human and canine heart cells. $A m \mathcal{F}$ Cardiol 1980;45:1189-95.

31 Seelig M. Cardiovascular consequences of magnesium deficiency and loss: pathogenesis, prevalence and manifestations: magnesium and chloride loss in refractory potassium repletion. $A m$ f Cardiol 1989;63:4-21G. 